\title{
ERK couples chronic survival of NK cells to constitutively activated Ras in lymphoproliferative disease of granular lymphocytes (LDGL)
}

\author{
Pearlie K Epling-Burnette*,1,2, Fanqi Bai ${ }^{1}$, Sheng Wei ${ }^{3}$, Pratima Chaurasia ${ }^{1}$, Jeffrey S Painter ${ }^{1}$, \\ Nancy Olashaw ${ }^{4}$, Andrew Hamilton ${ }^{5}$, Said Sebti ${ }^{6}$, Julie Y Djeu ${ }^{3}$ and Thomas P Loughran $\mathrm{Jr}^{7}$ \\ ${ }^{1}$ Hematologic Malignancies, Programs from the Department of Interdisciplinary Oncology, H Lee Moffitt Cancer Center and \\ Research Institute, University of South Florida, Tampa, FL 33612, USA; ${ }^{2}$ James A Haley Veterans' Administration Hospital, Tampa, \\ FL 33612, USA; ${ }^{3}$ Immunology, Programs from the Department of Interdisciplinary Oncology, $H$ Lee Moffitt Cancer Center and \\ Research Institute, University of South Florida, Tampa, FL 33612, USA; ${ }^{4}$ Molecular Oncology, Programs from the Department of \\ Interdisciplinary Oncology, H Lee Moffitt Cancer Center and Research Institute, University of South Florida, Tampa, FL 33612, \\ USA; ${ }^{5}$ Department of Chemistry, Yale University, New Haven, CT 06520, USA; ${ }^{6}$ Drug Discovery, Programs from the Department of \\ Interdisciplinary Oncology, $H$ Lee Moffitt Cancer Center and Research Institute, University of South Florida, Tampa, FL 33612, \\ USA; ${ }^{7}$ Penn State Cancer Institute, Penn State College of Medicine, Hershey, PA 17033, USA
}

Chronic NK lymphoproliferative disease of large granular lymphocytes (LDGL) is characterized by the expansion of activated $\mathrm{CD3}^{-}, \mathrm{CD16}^{+}$or $\mathrm{CD56}^{+}$lymphocytes. The mechanism of survival of NK cells from LDGL patients is unknown but may be related to antigenic stimulation. There is currently no standard effective therapy for LDGL, and the disease is characteristically resistant to standard forms of chemotherapy. We found evidence of constitutive activation of extracellular-regulated kinase (ERK) in NK cells from 13/13 patients with NK-LDGL (one patient with aggressive and 12 patients with chronic disease). Ablation of ERK activity by inhibitors or a dominant-negative form of MEK, the upstream activator of ERK, reduced the survival of patient NK cells. Ras was also constitutively active in patient NK cells, and exposure of cells to the Ras inhibitor FTI2153 or to dominantnegative-Ras resulted not only in ERK inhibition but also in enhanced apoptosis in both the presence and absence of anti-Fas. Therefore, we conclude that a constitutively active Ras/MEK/ERK pathway contributes to the accumulation of NK cells in patients with NK-LDGL. These findings suggest that strategies to inhibit this signaling pathway may be useful for the treatment of the NK type of LDGL.

Oncogene (2004) 23, 9220-9229. doi:10.1038/sj.onc.1208122 Published online 1 November 2004

Keywords: mitogen-activated protein kinase (MAPK); Ras; natural killer cells

*Correspondence: PK Epling-Burnette, James A Haley VA Hospital, 13000 Bruce B Downs Blvd, Research Bldg 2, Room 115, Tampa, FL 33612, USA; E-mail: burnetpk@moffitt.usf.edu

Received 26 May 2004; revised 19 July 2004; accepted 19 July 2004; published online 1 November 2004

\section{Introduction}

Large granular lymphocytes (LGLs) comprise $10-15 \%$ of normal peripheral blood mononuclear cells (PBMC) and are divided into two major lineages based on CD3 expression (Timonen et al., 1981). CD3 ${ }^{-}$LGLs are natural killer (NK) cells that do not express the CD3/Tcell receptor (TCR) complex or rearrange TCR genes. Additionally, these cells are capable of mediating nonMHC-restricted cytotoxicity (Lanier et al., 1986). CD3 ${ }^{+}$ LGLs are T cells, which express the CD3/TCR complex and rearrange TCR genes (Ritz et al., 1985). On the basis of the clonality and invasion of LGLs in marrow, spleen, and liver, we proposed the term 'LGL leukemia' for diseases characterized by increased numbers of circulating LGLs, which can arise for either CD3- or CD3 + LGLs (Loughran et al., 1985). Since it is difficult to determine clonality for NK cells, we and others have utilized the term 'lymphoproliferative disease of granular lymphocytes (LDGL)' to encompass NK LGL leukemias (Semenzato et al., 1987; Loughran, 1993).

NK-LDGL can have an aggressive or a chronic course. Features of aggressive NK-LDGL include large numbers of circulating NK cells, hepatosplenomegaly, and a median survival time of only a few months. Most cases of aggressive NK-LDGL occur in the Far East and are the result of EBV infection (Ohno et al., 1988; Taniwaki et al., 1990). Patients with chronic NK-LDGL have lymphocytosis that is often associated with bone marrow failure consisting of moderate anemia and/or neutropenia (Tefferi et al., 1994). Although the etiology of chronic NK-LDGL is not known, patients have high antibody titers to an envelope epitope (BA21) of HTLVI, suggesting that the disease may be related to an HTLV-like retroviral infection (Loughran et al., 1997).

Extracellular-regulated kinase (ERK) is a dual specificity kinase that regulates growth and differentiation in response to growth factors (Chang and Karin, 2001). Growth factors typically activate ERK by a multistep pathway. First, Grb-2/SOS associates with 
mitogen-activated receptors. Second, receptor-associated Grb-2/SOS recruits and activates Ras, a small farnesylated GTPase. Third, Ras activates Raf, which in turn activates ERK kinase 1 (MEK1). Fourth, MEK1 activates ERK by phosphorylating the TEY motif of ERK (Li et al., 1993). Through the activation of several antiapoptotic and cell cycle regulatory proteins, phosphorylated ERK elicits growth and survival signals.

Here, we show that NK cells derived from NK-LDGL patients possess cell surface and intracellular markers associated with in vivo activation. This finding suggests that survival signals associated with receptor engagement are active in these cells. Consistent with this premise, we demonstrate constitutive activation of ERK in NK cells from patients with NK-LDGL and the need for ERK activation for the survival of these cells.

\section{Results}

\section{Activated phenotype of freshly isolated NK cells from NK-LDGL patients}

We determined the expression patterns of CD56 and CD16 on enriched normal NK cells $(n=9)$ and NK cells derived from NK-LDGL patients $(n=11)$ by flow cytometry. Of the 13 patients examined, 12 had diagnoses consistent with chronic NK-LDGL (Table 1). All patients had NK lymphocytosis, and seven had associated bone marrow failure that consisted of either anemia (five out of seven), neutropenia (three out of seven) or both (NKLGL11 and NKLGL13).

As categorized on the basis of CD56 and CD16 expression patterns, normal cells isolated by negative selection contained one major subpopulation (R1) and four minor subpopulations (R2-R5) (Figure 1). Cells in the R1 subpopulation expressed large amounts of CD16 and coexpressed CD56 (CD16 high/CD56+). NK cells from NK-LDGL patients were more homogeneous than were normal NK cells, although no one subpopulation predominated (Figure 1 and Table 2). For example,

Table 1 Clinical characteristics of patients with NK-LDGL

\begin{tabular}{lrrrl}
\hline PT number & $W B C^{\mathrm{a}}$ & $A L C^{\mathrm{b}}$ & $N K$ & Symptoms $^{\mathrm{c}}$ \\
\hline NKLGL1 & 11000 & 7700 & 5082 & $\mathrm{~L} / \mathrm{N}$ \\
NKLGL2 & 8110 & 6001 & 2040 & $\mathrm{~L}$ \\
NKLGL3 & 9900 & 1485 & 713 & $\mathrm{~L}$ \\
NKLGL4 & 8220 & 6000 & 4440 & $\mathrm{~L} / \mathrm{A}$ \\
NKLGL5 & 6100 & 4636 & 3616 & $\mathrm{~L} / \mathrm{A}$ \\
NKLGL6 & 16400 & 11600 & 9164 & $\mathrm{~L}$ \\
NKLGL7 & 13390 & 12453 & 9340 & $\mathrm{UN}$ \\
NKLGL8 & 4200 & 882 & 838 & $\mathrm{Blastic}$ \\
NKLGL9 & 15900 & 9222 & 4150 & $\mathrm{~L}$ \\
NKLGL10 & 10700 & 5297 & 3496 & $\mathrm{~L}$ \\
NKLGL11 & 7100 & 2840 & 1704 & $\mathrm{~L} / \mathrm{A} / \mathrm{N}$ \\
NKLGL12 & 5700 & 3608 & 2995 & $\mathrm{~L} / \mathrm{A}$ \\
NKLGL13 & 10600 & 7345 & 5141 & $\mathrm{~L} / \mathrm{A} / \mathrm{N}$ \\
\hline
\end{tabular}

${ }^{\mathrm{a}} \mathrm{WBC}=$ white blood cell count. ${ }^{\mathrm{b}} \mathrm{ALC}=$ absolute lymphocyte count. 'Symptoms included lymphocytosis (L), anemia (A) and neutropenia $(\mathrm{N})$
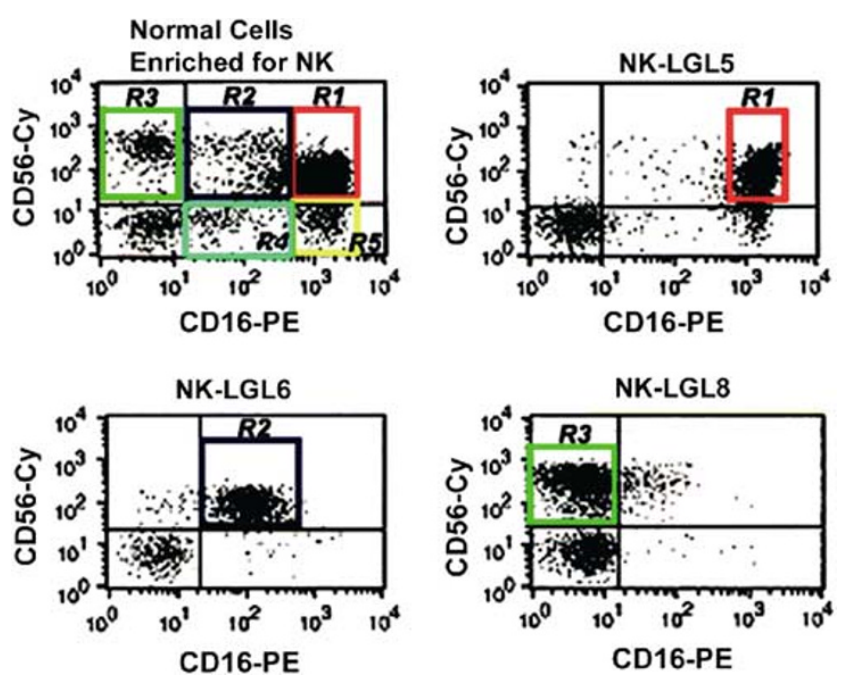

Figure 1 CD16 and CD56 expression on normal NK cells and NK cells from NK-LDGL patients. Flow cytometry dot plots are shown for normal and patient NK cells with CD56-Cy on the $y$ axis and CD16-PE on the $x$-axis. Subpopulations are: $\mathrm{R} 1=\mathrm{CD} 16$ high and CD56 positive (red), R2 = CD16 low and CD15 positive (blue), $\mathrm{R} 3=\mathrm{CD} 56$ positive and CD16 negative (green), $\mathrm{R} 4=\mathrm{CD} 16$ low and CD56 negative (aqua), R5 = CD16 high and CD56 negative (yellow)

nearly all NK cells from NKLGL6 were of the R2 subtype (Figure 1, bottom left), whereas nearly all NK cells from NKLGL8 were of the R3 subtype (Figure 1, bottom right). NKLGL8 had multiple genetic abnormalities with a diagnosis consistent with aggressive (blastic) LGL leukemia and was the only patient with NK cells limited to the R3 subpopulation. This subpopulation, which expressed CD56 in the absence of CD16, has been shown previously to represent a more immature NK phenotype (Husain et al., 2002).

We next compared the surface phenotype of normal NK cells and NK cells from patients using two-color flow cytometry to assess the co-expression of CD56, CD16 and the following proteins: HLA-DR, CD45RO, CD11c, CD95, CD11b, CD7, CD25, and CD69. CD45RA was detected on all normal and patient NK cells, whereas CD3 expression was $5 \%$ or less (data not shown). In agreement with published data, we found that the majority of $\mathrm{NK}$ cells from normal peripheral blood had the following phenotype: $\mathrm{CD}_{56}^{+}, \mathrm{CD}^{+} 6^{+}$, $\mathrm{CD}^{+}$(high, average MFI of $725 \pm 274$ ), HLA-DR negative, CD11b+ (high, average MFI $280 \pm 155$ ), and CD45RO negative (Table 2, average \pm s.d. of nine normal samples, NL NK) (Lima et al., 2002).

The surface phenotype of NK cells from NK-LDGL patients differed from that of normal NK cells in four respects. First, NK cells from four out of 12 patients expressed both CD45RO and CD45RA, whereas normal NK cells expressed CD45RA but not CD45RO (Table 2, NKLGL 3, 6, 7, and 8). Second, NK cells from eight out of 12 patients expressed substantially more HLA-DR than did normal cells (Table 2, NKLGL 1, 3, 4, 5, 6, 8, 10, and 12). Third, NK cells from all patients expressed less CD11b (MFI 112 \pm 48 ) than did normal 
Table 2 Immunophenotype of CD16 + or CD56 + NK cells from patients with NK-LDGL

\begin{tabular}{|c|c|c|c|c|c|c|c|c|c|c|c|}
\hline & \multirow{2}{*}{$\begin{array}{c}H L A-D R \\
(\%)\end{array}$} & \multirow{2}{*}{$\begin{array}{c}C D 45 R O \\
(\%)\end{array}$} & \multirow{2}{*}{$\begin{array}{c}C D 11 c \\
(\%)\end{array}$} & \multirow{2}{*}{$\begin{array}{c}C D 95 \\
(\%)\end{array}$} & \multicolumn{2}{|c|}{$C D 11 b$} & \multicolumn{2}{|c|}{$C D 7$} & \multirow{2}{*}{$\begin{array}{c}C D 25 \\
\%\end{array}$} & \multirow{2}{*}{$\begin{array}{c}C D 69 \\
\%\end{array}$} & \multirow{2}{*}{$\begin{array}{c}N K \\
\%\end{array}$} \\
\hline & & & & & $(\%)$ & $M F I$ & $(\%)$ & $(M F I)$ & & & \\
\hline NKLGL1 & $14^{\mathrm{a}}$ & 3 & 67 & 67 & 61 & 146 & 84 & 147 & 0 & 0 & 67 \\
\hline NKLGL2 & 3 & 3 & ND & 34 & 31 & 88 & 11 & 157 & 0 & 0 & 42 \\
\hline NKLGL3 & $36^{\mathrm{a}}$ & $27 "$ & 8 & 48 & 36 & 97 & 46 & 443 & 1 & 3 & 48 \\
\hline NKLGL4 & $7^{\mathrm{a}}$ & 1 & ND & 74 & 58 & 74 & 11 & 100 & 0 & 1 & 74 \\
\hline NKLGL5 & $39^{\mathrm{a}}$ & 6 & 76 & 78 & 73 & 46 & 72 & 128 & 0 & 2 & 78 \\
\hline NKLGL6 & $50^{\mathrm{a}}$ & $71^{a}$ & ND & 83 & 80 & 134 & 78 & 350 & 0 & 1 & 83 \\
\hline NKLGL7 & 5 & $25^{\mathrm{a}}$ & ND & 75 & 71 & 120 & 71 & 99 & 0 & 0 & 75 \\
\hline NKLGL8 & $92^{\mathrm{a}}$ & $90^{\mathrm{a}}$ & 93 & 95 & 83 & 110 & 25 & 81 & 0 & 2 & 95 \\
\hline NKLGL9 & 1 & 5 & 30 & 37 & 29 & 92 & 27 & 74 & 0 & 0 & 45 \\
\hline NKLGL10 & $41^{\mathrm{a}}$ & 6 & 8 & 56 & 31 & 91 & 34 & 118 & 0 & 1 & 58 \\
\hline NKLGL11 & 4 & 1 & 59 & 60 & 51 & 242 & 59 & 313 & 0 & 5 & 60 \\
\hline NKLGL12 & $33^{\mathrm{a}}$ & 3 & 81 & 83 & 48 & 101 & 30 & 90 & 0 & 5 & 83 \\
\hline Ave Patient & $27 \pm 22$ & $20 \pm 33$ & $53 \pm 25$ & $66 \pm 4$ & $54 \pm 10$ & $112 \pm 48^{\mathrm{a}}$ & $46 \pm 21$ & $175 \pm 122^{\mathrm{a}}$ & $0.1 \pm 0.2$ & $2 \pm 2$ & $67 \pm 16$ \\
\hline Ave NL NK & $1 \pm 1$ & $1 \pm 1$ & $2 \pm 1$ & $14 \pm 2$ & $16 \pm 3$ & $280 \pm 155$ & $17 \pm 1$ & $725 \pm 274$ & $0.4 \pm 4$ & $1 \pm 1$ & $18 \pm 7$ \\
\hline
\end{tabular}

Phenotype was determined by flow cytometry on PBMCs. Values indicate the percentage of CD16 + or CDS6 + cells that co-expressed the surface markers indicated in each column. Values shown as percentage positive cells from numbers obtained from the top right-hand quadrant of two-color dot plot flow cytometry graphs. MFI = mean fluorenscence intensity based on the relative expression of normal NK cells. NL NK = average

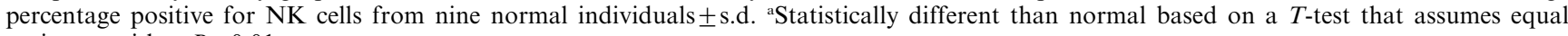
variances with a $P<0.01$

NK cells (MFI 280 \pm 155 ). Fourth, patients with NKLDGL had a greater percentage of CD11c-positive NK cells $(53 \%)$ than did normal individuals $(2 \%)$. Increased HLA-DR expression, co-expression of CD45RA and CD45RO, and reduced expression of CD11b were observed in a previous study of NK cells obtained from individuals with an acute viral infection (Lima et al., 2002). Therefore, the phenotypic pattern of surface markers expressed on NK cells from NK-LDGL patients is consistent with acute infection or activation.

During an acute viral infection, CD7 expression is maintained at levels similar to those of unactivated normal NK cells. In contrast, NK cells from NK-LDGL patients expressed less CD7 than did normal NK cells $(\mathrm{MFI}=175 \pm 122$ for patient NK cells and $725 \pm 274$ for normal NK cells; Table 2). Others have demonstrated subnormal amounts of CD7 in NK cells isolated from individuals with chronic viral infections or neoplasia (Lima et al., 2002). Thus, the immunophenotype of NK cells from NK-LDGL patients has combined features of both chronic and acute activation. This observation suggests that these cells are activated in vivo by persistent-active infection/activation.

Another measure of NK activation is sensitivity to CD16-mediated apoptosis. Therefore, we compared the sensitivity of normal NK cells and patient NK cells to apoptosis induced by CD16 ligation. In agreement with previous findings (Ortaldo et al., 1995), anti-CD16 antibody did not appreciably induce the apoptosis of normal NK cells unless they were pretreated with IL-2 (Figure 2). On the other hand, $20-40 \%$ of NK cells became apoptotic when exposed to anti-CD16 antibody (Figure 2). In agreement with the results of the immunophenotype analyses, the enhanced susceptibility of freshly isolated patient NK cells to CD16-mediated apoptosis suggests that these cells have been sensitized by prior activation in vivo.

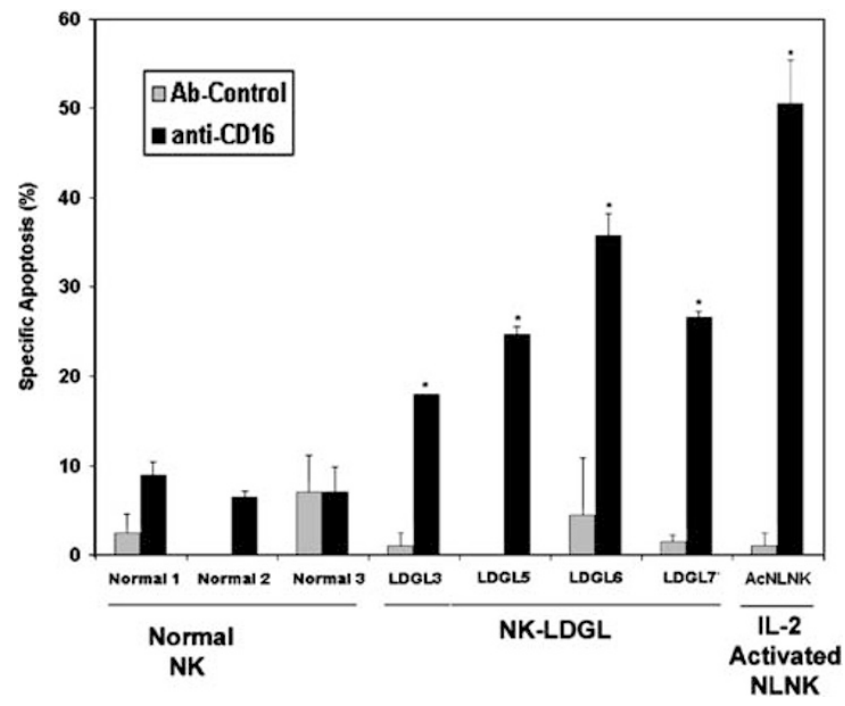

Figure 2 Sensitivity to CD16 (FC $\gamma$ RIII)-mediated apoptosis. Normal NK cells and NK cells from LDGL patients received no addition (gray bars) or $1 \mu \mathrm{g} / \mathrm{ml}$ anti-CD16 (black bars) for $48 \mathrm{~h}$. Some cultures of normal NK cells also received IL-2 for $24 \mathrm{~h}$ (AcNLNK). Apoptosis was determined as described in Material and methods. The percentage of apoptotic cells is shown \pm s.d. Asterisk (*) indicates a statistically significant increase from control using a paired $T$-test $(P<0.05)$

\section{Sensivity of NK-LDGL cells to Fas}

Large numbers of NK cells accumulate in the peripheral blood of patients with NK-LDGL by an unknown mechanism. Reduced apoptosis rather than increased cellular proliferation has been shown to contribute to the accumulation of $\mathrm{T}$ cells in patients with T-LGL leukemia (Lamy et al., 1998). Thus, we examined the cell cycle profile of NK cells from patients with NK-LDGL. Interestingly, we found that most of the NK cells are in 
the $\mathrm{G} 0 / \mathrm{G} 1$ phase of the cell cycle (data reproduced in four donors). This finding indicates that enhanced survival rather than increased proliferation accounts for the accumulation of NK cells in NK-LDGL patients. When exposed to IL-2, patient NK cells entered $\mathrm{S}$ phase after $72 \mathrm{~h}$ suggesting that the cells are capable of proliferation in response to growth factors (Figure 3A, panel b).

CD69 and CD25 (IL-2R $\alpha$ ) are transiently induced markers of activation after stimulation of NK cells that are associated with proliferation (Montagna et al., 1992; Ferlazzo et al., 2003). Additional findings from phenotype analysis include little if any CD69 and CD25 expression on NK cells from NK-LDGL patients (Table 2) consistent with the G0/G1 phase by cell cycle analysis.

Apoptosis mediated by the Fas receptor is thought to regulate numerous functions of the immune system including the peripheral elimination of activated T cells (Cohen et al., 1992; Miyawaki et al., 1992). The mechanism that removes activated normal NK cells from circulation is incompletely understood but may involve Fas receptors (Miyawaki et al., 1992; WatanabeFukunaga et al., 1992). Despite a slightly greater percentage of Fas receptor positive NK cells in patients than in normal (Table 2), both populations were equally and only minimally sensitive to apoptosis induced by Fas receptor ligation (Figure 3B). Exposure of normal (Ac NL) and patient NK cells to IL-2 for a short period of time increased their sensitivity to Fas-induced apoptosis (NK-LGL5, NK-LGL4, NK-LGL7, and NK-LGL10 (Figure 3B and data not shown). Collectively, the data shown in Figures 2 and 3 suggest that different signaling pathways control Fas-mediated apoptosis and CD16-mediated apoptosis. They also suggest that the failure to become sensitized to Fasmediated apoptosis may contribute to the accumulation of these cells after in vivo activation.

\section{Constitutively active ERK contributes to the survival of NK-LDGL cells}

Previous studies have demonstrated the need for ERK activity for the survival of antigen-activated $\mathrm{T}$ cells
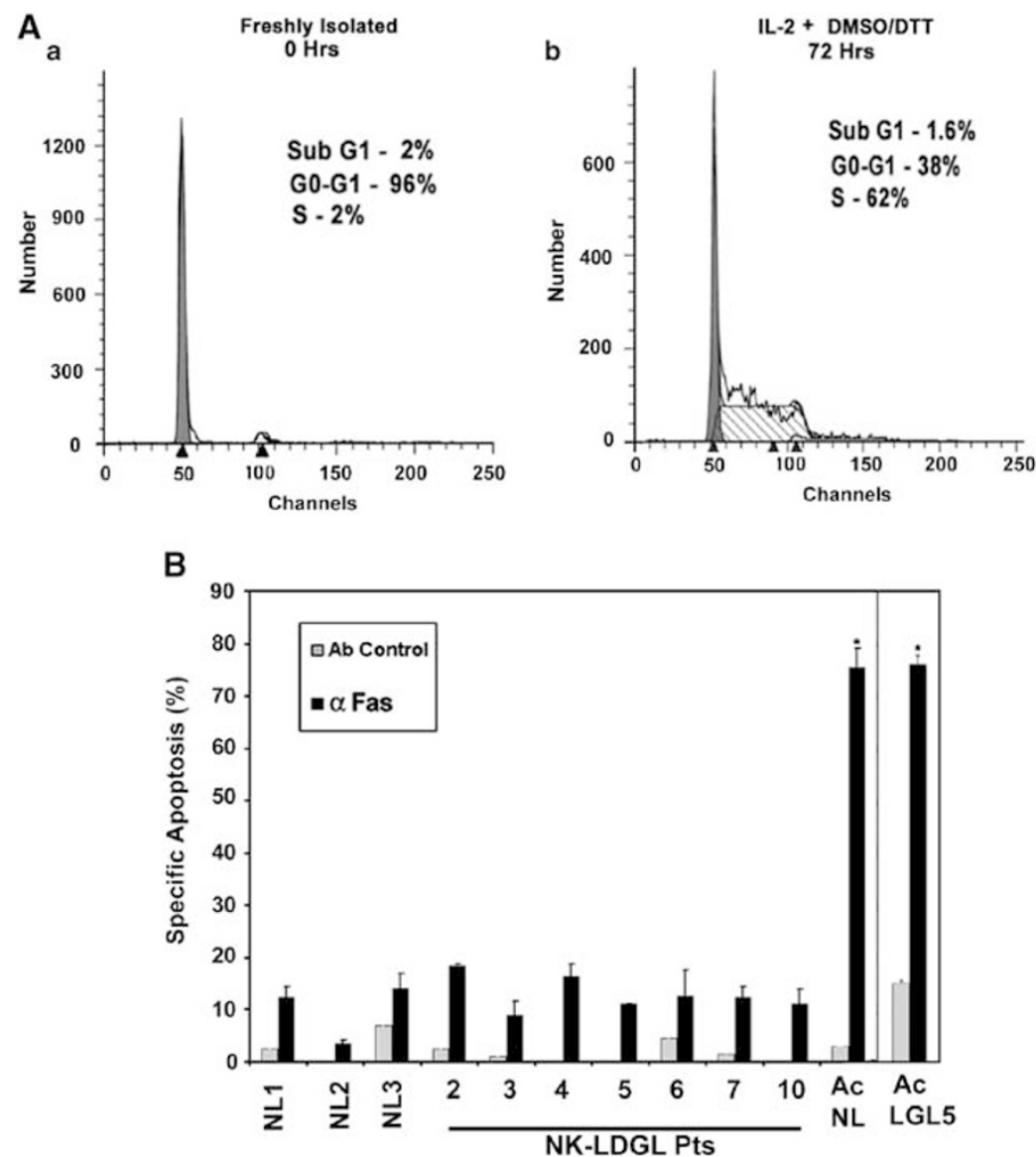

Figure 3 Cell cycle analysis and sensitivity to Fas. (A) Results of cell cycle analysis by PI staining of CD16 + (gated) NK cells from one characteristic patient (NKLGL5). (a) Cells were freshly isolated or (B) exposed to IL-2 for $72 \mathrm{~h}$. The percentages of sub-G1 (apoptotic), G0/G1, and S phase cells are shown. (b) Normal enriched NK cells (NL, 75-95\% NK) and PBMCs (containing 48-83\% NK) from NK-LDGL patients were exposed to $500 \mathrm{ng} / \mathrm{ml}$ anti-Fas agonistic antibody (CH11) (black bars) and $500 \mathrm{ng} / \mathrm{ml} \mathrm{purified}$ isotype control antibody (gray bars) for $48 \mathrm{~h}$. After activation with IL-2 (100 IU/ml for $72 \mathrm{~h}$ ), apoptosis of normal NK (AcNL) and patient NKLGL5 cells (AcNKLGL5) is shown as a positive control. The percentage of apoptotic cells is shown \pm s.d. as described in Material and methods. Asterisk $(*)$ indicates a statistically significant increase from control using a paired $T$-test $(P<0.05)$. IL-2 sensitization results were reproduced in three additional donors 
(Holmstrom et al., 2000) Little is known about the signaling pathways that control the survival of activated NK cells. To address this issue, we first monitored ERK activity in freshly isolated normal and patient NK cells by Western blot analysis using an antibody that specifically recognizes phosphorylated (and thus activate) ERK1 and ERK 2 (collectively referred to as ERK). We found that normal cells lacked detectable ERK activity (Figure 4a, lanes 1 and 3) and that exposure to IL-2 activated ERK (lanes 2 and 4). In contrast, patient NK cells exhibited ERK activity in the absence of IL-2 (lanes 5, 7, 9, 11, and 13). Incubation of patient cells with the MEK inhibitor PD98059 greatly reduced ERK activity without affecting total ERK levels (lanes 6, 8, 10, 12, and 14). Cells from two patients were incubated with PD98059 for increasing time to study the kinetic changes in pERK expression (Figure 4b). We found that the level of pERK was reduced within $12 \mathrm{~h}$. In cells from these two patients and one additional patient, the induction of apoptosis was not observed until $42 \mathrm{~h}$ (data not shown). These data suggest that the $\mathrm{MEK} / \mathrm{ERK}$ pathway is constitutively active in patient NK cells.

We next used MEK inhibitors to evaluate the need for ERK activity for the survival of NK cells from NKLDGL patients. When exposed to the MEK inhibitors PD98059 and U0126 in vitro, cells from patients exhibited enhanced apoptosis, as shown for a representative donor (Figure 4c; similar results were obtained for NK cells from four additional patients). Similar doses of these inhibitors had no effect on the survival of normal NK cells (Figure 4c; these data were reproduced in normal NK cells from two additional donors). In studies of PD98059 specificity and sensitivity, MEK1 and MEK2 were inhibited by $50 \mu \mathrm{M}$ but this dose failed to inhibit 18 other protein Ser/Thr kinases that included MEK homologues (Alessi et al., 1995). We found that maximal killing of NK cells from most patients occurred at a concentration of PD98059 of $50 \mu \mathrm{M}$ (Figure 4d). PBMC from NK-LGL8 could not be evaluated for sensitivity to the MEK inhibitors due to a high degree of spontaneous cell death at $42 \mathrm{~h}$.

Co-treatment of NK cells from NK-LDGL patients with PD98059 and anti-Fas antibody produced an additive increase in the percentage of apoptotic cells as compared with cells treated with anti-Fas antibody or PD98059 alone (Figure 5a). As determined by Western blot analysis and/or flow cytometry, PD98059 had no effect on the abundance of Fas receptor, Fas ligand, or ERK2 when added to NK cells from three patients (data not shown).

As another means of assessing the need for ERK activity for the survival of patient NK cells, we infected these cells with vaccinia virus encoding a dominantnegative form of MEK1 (DN-MEK1) (Wei et al., 1998). As negative controls, other sets of cells were mockinfected or infected with CD56. Infected cells were incubated with or without anti-Fas antibody for $12 \mathrm{~h}$ before harvest. At $12 \mathrm{~h}$, a similar (and low) percentage of mock-infected and CD56-control virus-infected cells were apoptotic (Figure 5b), and CD56 was detected in
CD56-infected cells (Figure 5c, panel b). These results demonstrate that the short infection time (12h) was sufficient for recombinant protein expression without activation of the viral lytic phase and direct viralinduced cell death.

Expression of DN-MEK1 ablated ERK activity without affecting total ERK levels (Figure 5c) and induced the apoptosis of patient NK cells (Figure 5b). DN-MEK1 in combination with anti-Fas antibody produced an additive increase in apoptosis. Similar results were obtained using cells from four additional patients (data not shown). These results suggest that the $\mathrm{MEK} / \mathrm{ERK}$ pathway provides signals that promote the survival of NK cells from patients with NK-LDGL.

\section{Ras-dependent ERK activity controls the survival of $N K$} cells from patients with NK-LDGL

In normal NK cells activated by direct target binding, ERK drives lytic activity and mobilization of granzyme B independently of Ras activity (Wei et al., 2000). However, Ras activation can be detected during direct target ligation (Wei et al., 2000). As shown in Figure 6a, Ras is also constitutively active in NK cells from LDGL patients. Ras activity was determined by GST-Raf-RBD precipitation, and IL-2-treated YT cells, which are NK leukemia cells, are shown as a positive control. Importantly, inhibition of Ras activity in patient NK cells by the farnesyltransferase inhibitor FTI2153 (Lerner et al., 1997; Sun et al., 1999) reduced ERK activity without affecting total amounts of ERK. This finding indicates that Ras contributes to ERK activation in NK cells from NK-LDGL patients.

The need for Ras activity for ERK activation in patient NK cells suggests that inhibition of Ras activity will reduce the survival of these cells, as does inhibition of ERK activity. This premise was confirmed by two methods. First, NK cells from NK-LDGL patients were treated with FTI2153 for $42 \mathrm{~h}$, and the percentage of apoptotic cells was determined. As shown in Figure 6b, FTI2153 effectively induced the apoptosis of patient NK cells. Addition of anti-Fas antibody to cells pretreated with FTI2153 resulted in an additive increase in apoptosis, as did treatment of cells with PD98059 (data not shown) and anti-Fas antibody (Figure 5a).

Second, NK cells from NK-LDGL patients were infected with vaccinia virus encoding a dominantnegative form of Ras (N-17 Ras). Whereas less than $20 \%$ of mock-infected cells or CD56-infected cells were apoptotic, almost 50\% of cells expressing N-17 Ras were apoptotic (Figure 6c). Moreover, addition of antiFas antibody to cells expressing N-17 Ras further increased the percentage of apoptotic cells. Western blot analysis showed overexpression of CD56 and Ras in cells infected with CD56 and N-17 Ras, respectively, and loss of ERK activity from cells infected with N-17 Ras (Figure 6d). These results show that Ras contributes to ERK activation and is required for the survival of NK cells from patients with NK-LDGL. 

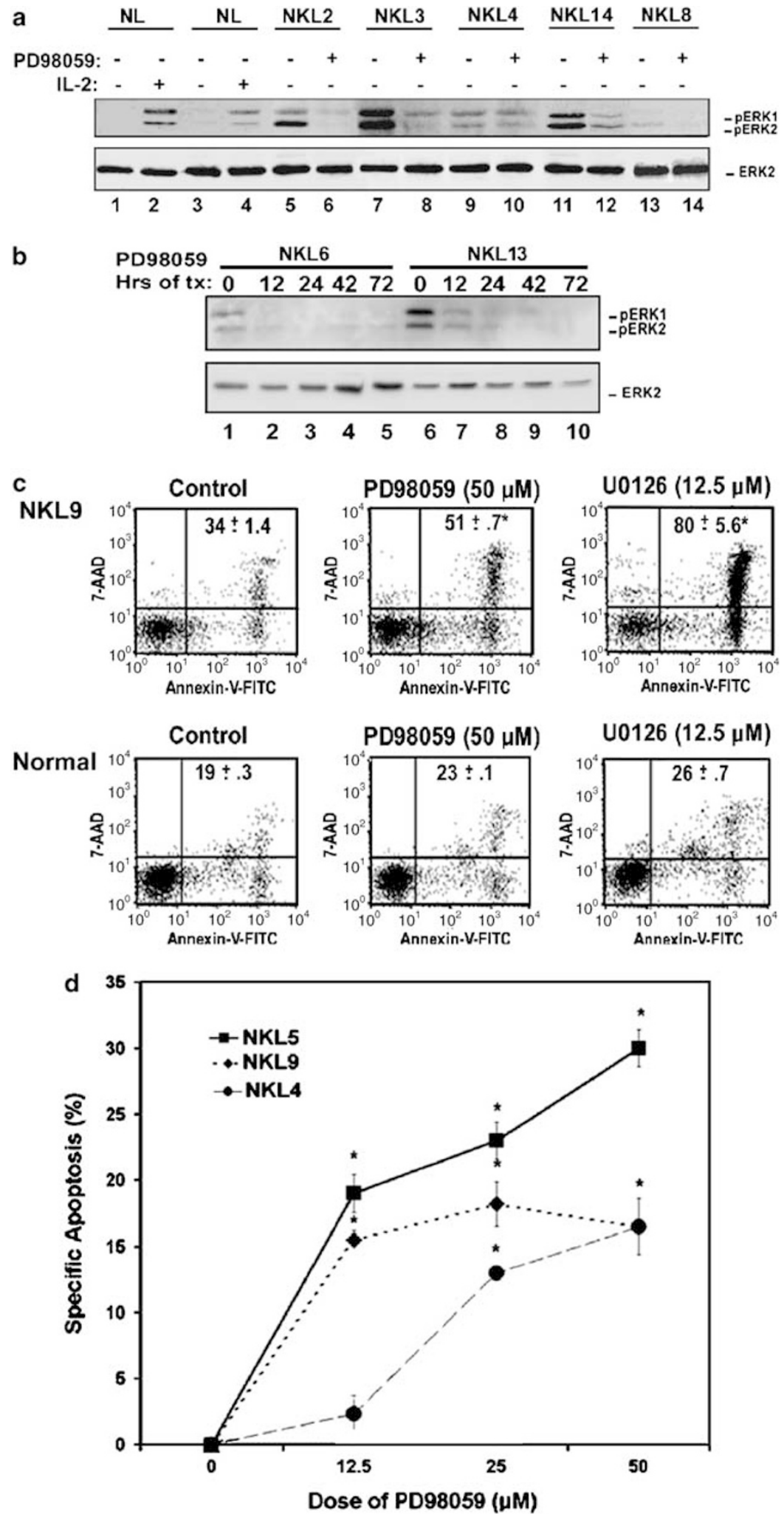

Figure 4 Patient NK cells express constitutively active MAPK that contribute to survival. (a) Normal enriched NK and patient PBMC with high percentages of NK cells were treated with $50 \mu \mathrm{M}$ PD 98059 or $100 \mathrm{IU} / \mathrm{ml} \mathrm{IL-2} \mathrm{for}(48 \mathrm{~h})$. (b) PBMC from two patients were treated with $50 \mu \mathrm{M}$ PD 98059 for 0 (freshly isolated), 12, 24, 42, and $72 \mathrm{~h}$. Amounts of activated and total ERK1/2 were determined by Western blotting. (c) Flow cytometry dot plot data of annexin-V-FITC binding (percentages in the lower right quadrants plus upper right quadrants are shown) and 7-AAD binding (upper right and upper left quadrants) in patient NK cells and normal NK cells treated with DMSO (vehicle control), PD98059 $(50 \mu \mathrm{M})$, or U0126 $(12.5 \mu \mathrm{M})$ for $42 \mathrm{~h}$. Asterisk $\left(^{*}\right)$ indicates a statistically significant increase from normal PBMC using a paired $T$-test $(P<0.05)$. (d) Patient NK cells were treated with $12.5,25$, or $50 \mu \mathrm{M}$ PD98059 for $42 \mathrm{~h}$. The bar graph shows the amount of apoptosis. Asterisk $(*)$ indicates a statistically significant increase from control using a paired $T$-test $(P<0.05)$ 


\section{Discussion}

NK-LDGL is a lymphoproliferative disease characterized by increased numbers of circulating NK cells. Since NK cells from such patients are not actively cycling, we hypothesize that the accumulation of these cells is due to activation of survival pathways leading to apoptosis
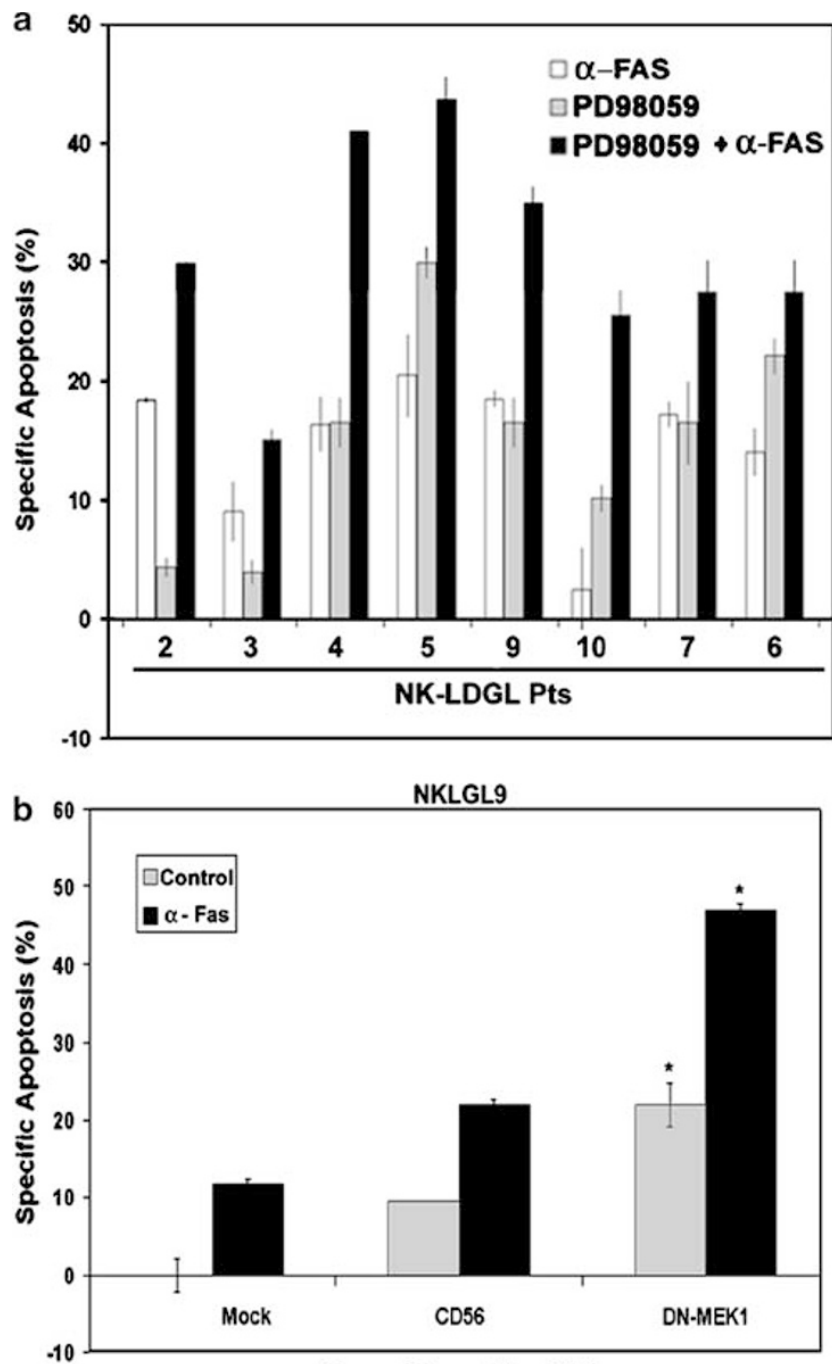

Type of Recombinant W

C

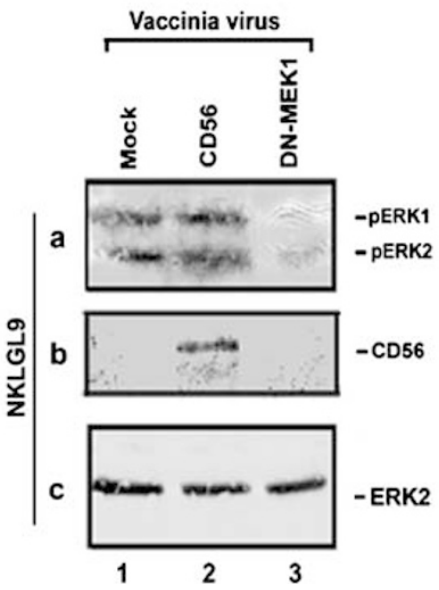

resistance. There is currently no standard effective therapy for either the chronic form or aggressive form of LDGL, and these diseases are characteristically resistant to standard forms of chemotherapy. Determination of the molecular signals that promote the survival of patient NK cells will facilitate the design of effective targeted drug therapies and will also provide insights into the mechanisms that regulate the survival of activated normal NK cells.

Here, we report that NK cells from a large majority of patients with NK-LDGL express constitutively active ERK and undergo apoptosis in response to $\mathrm{MEK} / \mathrm{ERK}$ inhibition. The mechanisms leading to constitutive ERK activation are unknown but may be related to dysregulated $\mathrm{NK}$ receptor stimulation. We and others have found that activation of normal NK cells by target binding or by ligation of activation receptors leads to ERK activation (Jiang et al., 2000; Zingoni et al., 2000; McVicar and Burshtyn, 2001; Djeu et al., 2002). Interestingly, we recently reported a skewed repertoire of NK receptor expression that consisted of predominately activating receptors in this cohort of patients (Epling-Burnette et al., 2004). We previously found that ERK-dependent tumorcidal function was Ras-independent. In fact, this function required the activation of another GTPase, Rac that activated PAK and ERK (Wei et al., 2000). Therefore, it is conceivable that there may be different upstream signaling components that lead to ERK activation in NK cells when comparing survival signaling to cytolytic activity. Cellular recognition and release of granules into a susceptible target requires approximately $15 \mathrm{~min}$ and is independent of transcriptional activity. In contrast, Ras may induce a more sustained ERK signal that is required for either enhanced protein stability of antiapoptotic proteins such as those of the Bcl-2 family or in the regulation of transcription factors and other proteins involved in survival. The exact mechanisms and targets of ERK-mediated survival signals are not known. It is known that $\mathrm{MEK} / \mathrm{ERK}$ signaling suppresses Fasmediated apoptosis in activated $\mathrm{T}$ cells by inhibiting DISC-mediated activation of caspase 8. (Holmstrom et al., 2000; Tran et al., 2001; He et al., 2002; Soderstrom et al., 2002). Other antiapoptotic proteins such as Bcl-2 and BAD are regulated by activated MAPK

Figure 5 MEK inhibition leads to increase Fas sensitivity. (a) Bar graph of percent apoptosis of patient NK cells treated with PD98059 $(50 \mu \mathrm{M})$, anti-Fas agonistic antibody $(\mathrm{CH} 11)(500 \mathrm{ng} / \mathrm{ml})$, or both for $42 \mathrm{~h}$. Apoptosis was calculated using the medium control as background as described in the Materials and methods section. There was no difference the percentage of apoptotic cells in medium with and without DMSO. (b) Patient NK cells were mockinfected (mock), infected with CD56 control virus (CD56), or infected with recombinant vaccinia virus expressing DN-MEK1. Percentage of apoptosis was calculated \pm s.d. as described in Material and methods after the cells were treated with control antibody (IgM) alone (Con, gray bars) or anti-Fas agonistic antibody $\mathrm{CH} 11$ (anti-Fas) for $12 \mathrm{~h}$ after viral infection. Asterisk (*) indicates a statistically significant increase from mock-infected cells using a paired $T$-test $(P<0.05)$. (c) Lysates were examined by Western blot with antibodies to phosphorylated ERK (pERK1/2), CD56, and total ERK2 
a

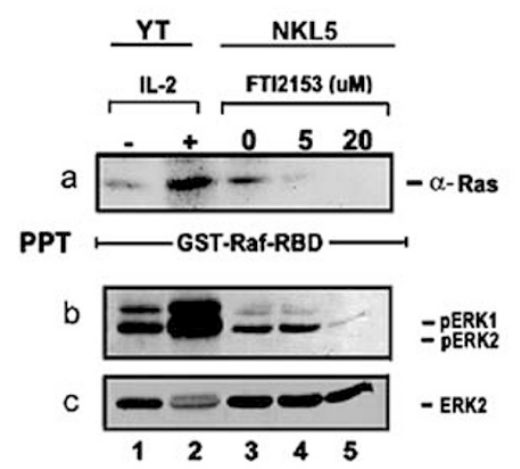

b

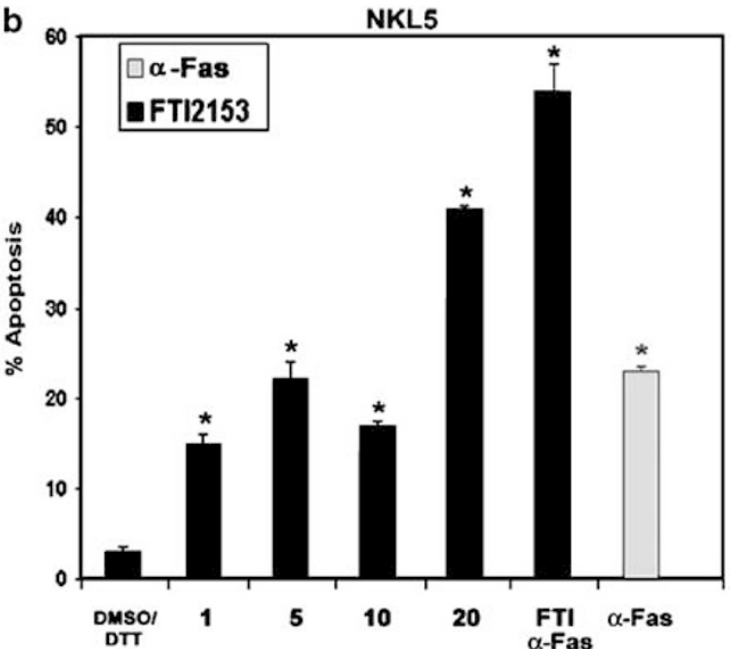

c

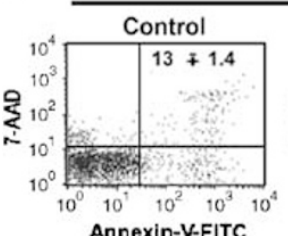

NKL10
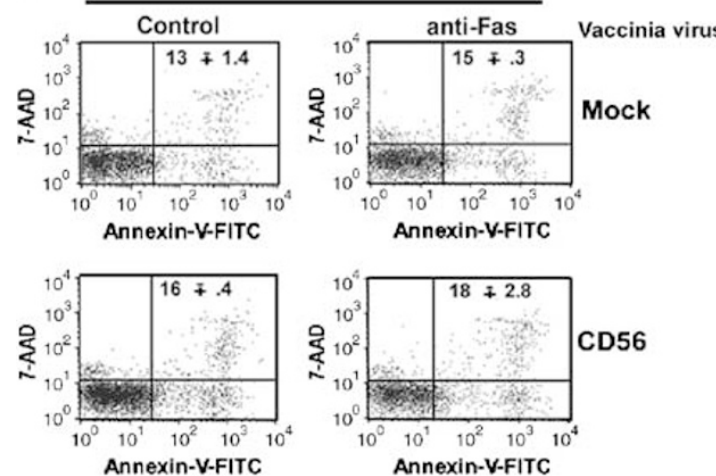

CD56
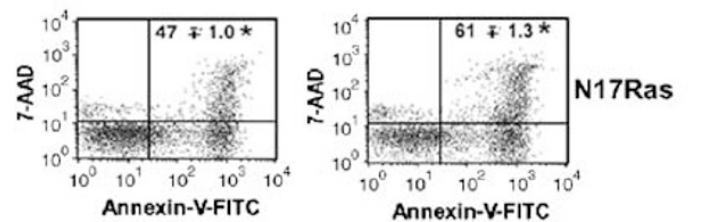

d

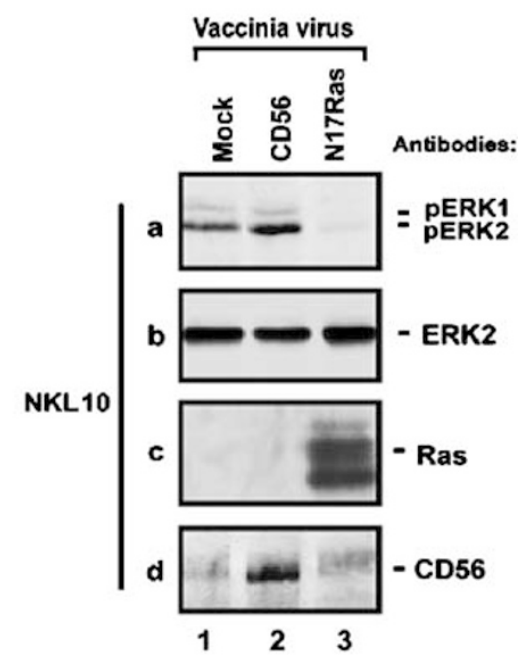

Figure 6 Ras Inhibition blocks ERK activation and induces apoptosis in NK cells from patients with NK-LDGL. (a) The NK leukemia cell line YT was incubated with medium with or without IL-2 for $30 \mathrm{~min}$. Freshly isolated patient PBMCs were treated with DMSO/DTT (0) or FTI2153 at 5 and $20 \mu \mathrm{M}$ for $42 \mathrm{~h}$. (a) GST-Raf-RBD-precipitated proteins were examined for the expression of activated Ras using the pan-Ras antibody. (b, c) Whole-cell lysates were analysed for activated ERK (pERK1/2) or total ERK2 expression. All these experiments were reproduced in three additional patients. (b) Simultaneous experiments to examine apoptosis were setup using patient PBMCs with high percentages of NK cells treated with FTI2153 at 1, 5, 10, or $20 \mu \mathrm{M}$ for $42 \mathrm{~h}$; the drug vehicle DMSO/DTT used as a control (black bars). Patient PBMCs also received FTI2153 plus anti-Fas ( $20 \mu \mathrm{M}$ and $500 \mathrm{ng} / \mathrm{ml}$, respectively) or anti-Fas alone (gray bar). The percentage of apoptotic cells was determined. Asterisk $(*)$ indicates a statistically significant increase from control using a paired $T$-test $(P<0.05)$. (c, d) Patient PBMCs were mock-infected (mock), infected with CD56 control virus (CD56), or infected with recombinant vaccinia virus expressing dominant-negative Ras (N-17Ras). Cells were then treated with or without anti-Fas antibody (CH11) for $12 \mathrm{~h}$. (c) Flow cytometry dot plots of annexin-V-FITC and 7-AAD binding (percentage positive indicated by adding the percentages in the upper right and lower right quadrants \pm s.d.). Asterisk $(*)$ indicates a statistically significant increase from IgM or $\mathrm{CH} 11$ treatment in mock-infected cells using a paired $T$-test $(P<0.05)$. (d) Western blots of cell lysates that detect activated MAPK (pERK1/2, top panel), total MAPK (ERK2), total Ras, and CD56. These experiments were reproduced in two additional donors

and may contribute to survival in these NK cells. (Scheid et al., 1999).

We demonstrate here that NK cells from patients with NK-LDGL have an activated phenotype, and it has been proposed that an unknown, perhaps viral, antigen is responsible for expansion of pre-LDGL NK populations (Loughran et al., 1997). Although these patients are not infected with prototypical HTLV-I or II, sera from most patients react with the BA21 epitope of the p21env protein of HTLV-I (Loughran et al., 1994, 1997; Perzova et al., 2000). These data suggest that patients with NK-LDGL have been exposed to a protein homologous to BA21, perhaps by infection with a novel retrovirus. Therefore, it is of interest that a synthetic peptide (CKS-17) derived from the p21env of HTLV-I induces ERK activation (Takahashi et al., 2001). 
The data presented here show that a Ras/MEK/ERKdependent pathway contributes to the abnormal survival in NK cells from patients with NK-LDGL. In vitro inhibition of both Ras activity and MEK activity led to increased apoptosis in patient NK cells. The results of preclinical and clinical studies show that FTIs typically inhibit the proliferation of tumor cells without appreciably affecting their survival (Lerner et al., 1995; Sun et al., 1999). Thus, we found that FTIs uniquely affect quiescent cells with an activated phenotype. New therapeutic strategies are greatly needed, particularly for the aggressive form of NK leukemia. Results of these studies identify the Ras/MEK/ERK signaling pathway as an important molecular target for drug discovery in NK-LDGL and NK leukemia.

\section{Materials and methods}

\section{Patients and preparation of PBMC}

All patients met the clinical criteria of NK-LDGL with increased numbers of $\mathrm{CD}^{-} / \mathrm{CD}^{2} 6^{+}$or $\mathrm{CD}^{-} / \mathrm{CD} 16^{+}$lymphocytes. All patients with chronic disease $(n=11)$ were clinically stable and untreated at the time of sample acquisition. One patient with aggressive NK leukemia was studied at the time of diagnosis, prior to the initiation of therapy. Informed consents were signed by all patients prior to the use of their cells for these experiments. Buffy coats from nine normal donors were also obtained from the Southwest Florida blood bank. PBMCs were isolated from buffy coats by FicollHypaque gradient separation, as previously described (EplingBurnette et al., 2001). In some experiments, enriched normal NK cells were isolated from buffy coats by negative selection using RosetteSep ${ }^{\mathrm{TM}}$, as recommended by the manufacturer (Stem Cell Separation Systems, Vancouver, BC, Canada). We found that these cells contained $\leqslant 1 \% \quad \mathrm{~T}$ cells, $\leqslant 1 \%$ monocytes and neutrophils, and $\leqslant 4 \% \quad B$ cells. Where indicated, normal and patient NK cells were activated by treatment with $100 \mathrm{U} / \mathrm{ml}$ of IL-2 for 1-3 days.

\section{Immunophenotyping}

Paired normal and patient NK cells were analysed by two-color flow cytometry using CyChrome-conjugated CD56 (clone B159, BD Biosciences, San Diego, CA, USA) or CD16-fluorescein isothiocyanate (FITC) in combination with the following phycoerythrin (PE)-conjugated antibodies: anti-HLA-DR, anti-CD45RO, anti-CD11c, anti-CD95, antiCD11b, anti-CD7, anti-CD25, and anti-CD69 (all from BD Biosciences). Data acquisition and analysis was carried out on a FACScan flow cytometer (BD Biosciences) using the Cell Quest software program. Forward scatter (FSC) and side scatter (SSC) plots were used to identify the lymphocyte population and a total of $1 \times 10^{5}$ total events were collected. In all samples analysed, the percentage of NK cells within the lymphocyte population was determined by their CD56 and CD16 staining pattern. The percentages of CD56 or CD16 positive cells coexpressing the surface marker of interest (double positive) was determined from the upper right hand

\section{References}

Alessi DR, Cuenda A, Cohen P, Dudley DT and Saltiel AR. (1995). J. Biol. Chem., 270, 27489-27494.

Chang L and Karin M. (2001). Nature, 410, 37-40. quadrant of the flow cytometry dot plot. Mean fluorescence intensity (MFI) of patient NK cells relative to normal NK cells was also determined.

\section{Apoptosis assay}

To measure apoptosis, we performed two-color flow cytometry with 7-amino-actinomycin D (7-AAD; Calbiochem-Novabiochem) and annexin-V-FITC (5 $\mu \mathrm{l}$; BD Pharmingen Transduction Labs., Place), as previously described (Epling-Burnette et al., 2001). Cell viability was also determined by trypan blue staining and quantitated by labeling with a DNA intercolating dye, 7-AAD, and analysis by flow cytometry. The percent specific apoptosis was calculated using the following formula:

$((\%$ annexin-V-FITC positive in the assay well)$(\%$ annexin-V-FITC positive in the control well $) \times 100) /(100-$ ( $\%$ annexin-V-FITC positive in the control well))

\section{Cell lysis and Western immunoblotting}

Cells were lysed in cell lysis buffer and Western blot analysis was performed as previously described (Epling-Burnette et al., 2001). Antibodies were obtained from the following sources and used at the dilutions recommended by the manufacturer: antiphosphorylated (active) MAPK (ERK1 and 2, New England Biolabs), antitotal MAPK, and antipanRas all from BD Biosciences, and anti- $\beta$-actin (Sigma Chemical Co.). Antibody detection was performed by standard ECL techniques as recommended by the manufacturer (Amersham Pharmacia Biotech). Protein quantification was performed by densitometry using the Image Quant program (Molecular dynamics, Sunnyvale, CA, USA).

\section{Detection of activated Ras}

To determine the constitutive activation status of Ras in patient NK cells, we used the GST-Raf-RBD-pGEX-KT construct expressed in bacteria to precipitate GTP-bound Ras with glutathione-agarose beads, as previously decribed (Wei et al., 2000). Western blot analysis was performed to detect precipitated activated Ras (anti-panRas antibody; BD Pharmingen Transduction Labs).

\section{Vaccinia virus gene delivery}

Recombinant vaccinia viruses were constructed using the vector, pSC11, as previously described (Wei et al., 2000). For infection, patient NK cells were incubated at a multiplicity of infection of 3:1 with either CD56 control virus, DN-MEK1, or DN-Ras (N17Ras) for $2 \mathrm{~h}$. The infected cells were then cultured in the presence of medium alone (control) or medium with $500 \mathrm{ng} / \mathrm{ml}$ of anti-Fas agonistic antibody (CH11) for an additional $12 \mathrm{~h}$. Cells were analysed for apoptosis using annexin-V-FITC/7-AAD by flow cytometry.

\section{Acknowledgements}

This work was supported by the following grants: Veterans' Administration Hospital and National Institute of Health Grants AI056213, CA90633, CA83146, CA83947, and CA94872. We would like to thank the Flow Cytometry Core and Molecular Imaging Core Facilities at $\mathrm{H}$ Lee Moffitt Cancer Center for assistance with data analysis and preparation of this manuscript.

Cohen JJ, Duke RC, Fadok VA and Sellins KS. (1992). Annu.

Rev. Immunol., 10, 267-293.

Djeu JY, Jiang K and Wei S. (2002). Clin. Cancer Res., 8, 636-640. 
Epling-Burnette PK, Liu JH, Catlett-Falcone R, Turkson J, Oshiro M, Kothapalli R, Li Y, Wang JM, Yang-Yen HF, Karras J, Jove R and Loughran Jr TP. (2001). J. Clin. Invest., 107, 351-362.

Epling-Burnette PK, Painter JS, Chaurasia P, Bai F, Wei S, Djeu JY and Loughran TP. (2004). Blood, 103, 3431-3439.

Ferlazzo G, Morandi B, D’Agostino A, Meazza R, Melioli G, Moretta A and Moretta L. (2003). Eur. J. Immunol., 33, 306-313.

He T, Stepulak A, Holmstrom TH, Omary MB and Eriksson JE. (2002). J. Biol. Chem., 277, 10767-10774.

Holmstrom TH, Schmitz I, Soderstrom TS, Poukkula M, Johnson VL, Chow SC, Krammer PH and Eriksson JE. (2000). EMBO J., 19, 5418-5428.

Husain Z, Alper CA, Yunis EJ and Dubey DP. (2002). Immunology, 106, 373-380.

Jiang K, Zhong B, Gilvary DL, Corliss BC, Hong-Geller E, Wei S and Djeu JY. (2000). Nat. Immunol., 1, 419-425.

Lamy T, Liu JH, Landowski TH, Dalton WS and Loughran Jr TP. (1998). Blood, 92, 4771-4777.

Lanier LL, Cwirla S, Federspiel N and Phillips JH. (1986). J. Exp. Med., 163, 209-214.

Lerner EC, Qian Y, Blaskovich MA, Fossum RD, Vogt A, Sun J, Cox AD, Der CJ, Hamilton AD and Sebti SM. (1995). J. Biol. Chem., 270, 26802-26806.

Lerner EC, Zhang TT, Knowles DB, Qian Y, Hamilton AD and Sebti SM. (1997). Oncogene., 15, 1283-1288.

Lima M, Almeida J, dos Anjos Teixeira M, Queiros ML, Justica B and Orfao A. (2002). Blood Cells Mol. Dis., 28, $181-190$

Li N, Batzer A, Daly R, Yajnik V, Skolnik E, Chardin P, Bar-Sagi D, Margolis B and Schlessinger J. (1993). Nature, 363, 85-88.

Loughran Jr TP. (1993). Blood, 82, 1-14.

Loughran Jr TP, Hadlock KG, Yang Q, Perzova R, Zambello R, Semenzato G, Foung SK and Poiesz BJ. (1997). Blood, 90, 1977-1981.

Loughran Jr TP, Kadin ME, Starkebaum G, Abkowitz JL, Clark EA, Disteche C, Lum LG and Slichter SJ. (1985). Ann. Intern. Med., 102, 169-175.

Loughran Jr TP, Sherman MP, Ruscetti FW, Frey S, Coyle T, Montagna RA, Jones B, Starkebaum G and Poiesz BJ. (1994). Leuk. Res., 18, 423-429.

McVicar DW and Burshtyn DN. (2001). Sci. STKE, 2001, RE1.

Miyawaki T, Uehara T, Nibu R, Tsuji T, Yachie A, Yonehara $\mathrm{S}$ and Taniguchi N. (1992). J. Immunol., 149, 3753-3758.
Montagna D, Moretta A, Marconi M, Mingrat G, Gasparoni A, Giarola M and Maccario R. (1992). Biol. Neonate, 62, 385-394.

Ohno T, Kanoh T, Arita Y, Fujii H, Kuribayashi K, Masuda T, Horiguchi Y, Taniwaki M, Nosaka T, Hatanaka M and Uchino I. (1988). Cancer, 62, 1918-1927.

Ortaldo JR, Mason AT and O'Shea JJ. (1995). J. Exp. Med., 181, 339-344.

Perzova RN, Loughran TP, Dube S, Ferrer J, Esteban E and Poiesz BJ. (2000). Br. J. Haematol., 109, 64-70.

Ritz J, Campen TJ, Schmidt RE, Royer HD, Hercend T, Hussey RE and Reinherz EL. (1985). Science, 228, 1540-1543.

Scheid MP, Schubert KM and Duronio V. (1999). J. Biol. Chem., 274, 31108-31113.

Semenzato G, Pandolfi F, Chisesi T, De Rossi G, Pizzolo G, Zambello R, Trentin L, Agostini C, Dini E, Vespignani M, Cafaro A, Pasqualetti D, Giubellino C, Migone N and Foa R. (1987). Cancer, 60, 2971-2978.

Soderstrom TS, Poukkula M, Holmstrom TH, Heiskanen KM and Eriksson JE. (2002). J. Immunol., 169, 2851-2860.

Sun J, Blaskovich MA, Knowles D, Qian Y, Ohkanda J, Bailey RD, Hamilton AD and Sebti SM. (1999). Cancer Res., 59, 4919-4926.

Takahashi A, Day NK, Luangwedchakarn V, Good RA and Haraguchi S. (2001). J. Immunol., 166, 6771-6775.

Taniwaki M, Tagawa S, Nishigaki H, Horiike S, Misawa S, Shimazaki C, Maekawa T, Fujii H, Kitani T and Abe T. (1990). Am. J. Hematol., 33, 32-38.

Tefferi A, Li CY, Witzig TE, Dhodapkar MV, Okuno SH and Phyliky RL. (1994). Blood, 84, 2721-2725.

Timonen T, Ortaldo JR and Herberman RB. (1981). J. Exp. Med., 153, 569-582.

Tran SE, Holmstrom TH, Ahonen M, Kahari VM and Eriksson JE. (2001). J. Biol. Chem., 276, 16484-16490.

Watanabe-Fukunaga R, Brannan CI, Copeland NG, Jenkins NA and Nagata S. (1992). Nature, 356, 314-317.

Wei S, Gamero AM, Liu JH, Daulton AA, Valkov NI, Trapani JA, Larner AC, Weber MJ and Djeu JY. (1998). J. Exp. Med., 187, 1753-1765.

Wei S, Gilvary DL, Corliss BC, Sebti S, Sun J, Straus DB, Leibson PJ, Trapani JA, Hamilton AD, Weber MJ and Djeu JY. (2000). J. Immunol., 165, 3811-3819.

Zingoni A, Palmieri G, Morrone S, Carretero M, Lopez-Botel M, Piccoli M, Frati L and Santoni A. (2000). Eur. J. Immunol., 30, 644-651. 